\title{
Differential effects of cognitive reserve and brain reserve on cognition in Alzheimer disease
}

Colin Groot, MSc, Anna C. van Loenhoud, MSc, Frederik Barkhof, MD, PhD, Bart N.M. van Berckel, MD, PhD, Teddy Koene, MSc, Charlotte C. Teunissen, MD, PhD, Philip Scheltens, MD, PhD, Wiesje M. van der Flier, PhD, and Rik Ossenkoppele, PhD

Neurology ${ }^{\circledR}$ 2018;90:e149-156. doi:10.1212/WNL.0000000000004802

\begin{abstract}
\section{Objective}

To examine cross-sectional effects of cognitive reserve (CR) and brain reserve (BR) on cognition across the spectrum of Alzheimer disease $(\mathrm{AD})$.

\section{Methods}

We included $663 \mathrm{AD}$ biomarker-positive participants with dementia (probable $\mathrm{AD}, \mathrm{n}=462$ ) or in the predementia stages (preclinical/prodromal $\mathrm{AD}, \mathrm{n}=201$ ). Education was used as a proxy of $\mathrm{CR}$ and intracranial volume as a proxy of BR. Cognition was assessed across 5 domains (memory, attention, language, visuospatial, and executive functions). We performed multiple linear regression models to examine effects of $\mathrm{CR}$ and $\mathrm{BR}$ on cognitive domain $Z$ scores, adjusted for cerebral atrophy. Furthermore, we assessed differences in effects according to disease stage and across degrees of total reserve using a 4-level variable (high $\mathrm{CR} /$ high $\mathrm{BR}$, high $\mathrm{CR} /$ low BR, low $\mathrm{CR} /$ high $\mathrm{BR}$, and low $\mathrm{CR} /$ low BR).
\end{abstract}

\section{Results}

We found positive, independent effects of both CR and BR across multiple cognitive domains. Stratification for disease stage showed that effects of $\mathrm{CR}$ on attention and executive functioning were greater in predementia than in dementia $(\beta=0.39$ vs $\beta=0.21$ [Welch $t=2.40, p<0.01$ ] and $\beta=0.46$ vs $\beta=0.26[t=2.83, p<0.01]$ ). Furthermore, we found a linear trend for better cognitive performance in all domains in the high $\mathrm{CR} /$ high $\mathrm{BR}$ group, followed by high $\mathrm{CR} /$ low $\mathrm{BR}$, low $\mathrm{CR} /$ high $\mathrm{BR}$, and then low $\mathrm{CR} /$ low $\mathrm{BR}(p$ for trend $<0.05)$.

\section{Conclusions}

$\mathrm{CR}$ and $\mathrm{BR}$ both independently mitigate cognitive symptoms in $\mathrm{AD}$. The positive effect of $\mathrm{CR}$ is most strongly expressed in the predementia stages and the additive effects of high CR and BR are most beneficial.

\author{
Correspondence \\ Colin Groot \\ c.groot3@vumc.nl
}




\section{Glossary}

$\mathbf{A} \boldsymbol{\beta}=\boldsymbol{\beta}$-amyloid $\mathbf{A D}=$ Alzheimer disease $\mathbf{B R}=$ brain reserve; $\mathbf{C R}=$ cognitive reserve $\mathbf{I C V}=$ intracranial volume; $\mathbf{M C I}=$ mild cognitive impairment; MMSE = Mini-Mental State Examination; NIA-AA = National Institute on Aging and Alzheimer's Association; SCD = subjective cognitive decline.

Neuropathologic and biomarker studies in patients with Alzheimer disease $(\mathrm{AD})$ have revealed remarkable interindividual differences in the level of cognitive function at a comparable neuropathologic burden. ${ }^{1-3}$ To account for these clinicopathologic discrepancies, the concept of reserve has been proposed. $^{4-6}$ Reserve describes the capacity to preserve cognitive function in the presence of neuropathology and can be divided into 2 components: cognitive reserve (CR) and brain reserve (BR). ${ }^{4} \mathrm{CR}$ is thought to act by recruiting alternate neural networks or utilizing existing networks more efficiently to cope with neuropathologic changes, and is often estimated using educational attainment. ${ }^{4-6}$ When matched for clinical disease severity, patients with $\mathrm{AD}$ with higher education have more advanced levels of neuropathology, ${ }^{7,8}$ indicating that individuals with greater $\mathrm{CR}$ can tolerate greater neuropathologic burden. BR represents a higher quantity of neural resources acting as a buffer that enables the brain to better tolerate emerging neuropathology, and is typically operationalized by intracranial volume (ICV) in human neuroimaging studies. ${ }^{9-11}$ ICV increases during development ${ }^{12}$ but remains largely stable with neurodegeneration due to chronologic aging or $\mathrm{AD},{ }^{13}$ and has been shown to act as a resilience factor against clinical deterioration in the presence of $\mathrm{AD}$ pathology. ${ }^{9-11}$ In the present study of $\mathrm{AD}$ biomarker-positive (preclinical, prodromal, and dementia) participants, we examine the independent and additive effects of CR and BR on memory, attention, visuospatial, language, and executive functions, while controlling for the degree of neurodegeneration as measured by cerebral atrophy.

\section{Methods}

\section{Participants}

In this cross-sectional study, we included 663 participants with positive $\mathrm{AD}$ biomarkers. The sample was selected from the Amsterdam Dementia Cohort ${ }^{14}$ and consisted of patients who visited the memory clinic of the VU University Medical Center in Amsterdam between January 2008 and December 2015 who consented to have their data used for research. All participants underwent standardized dementia screening including medical history, informant-based history, physical and neurologic examinations, lumbar puncture, brain MRI, and neuropsychological testing. Clinical diagnosis of probable $\mathrm{AD}$ or mild cognitive impairment $(\mathrm{MCI})$ due to $\mathrm{AD}$ was established by consensus in a multidisciplinary team according to National Institute on Aging and Alzheimer's Association (NIA-AA) criteria. ${ }^{15,16}$ A diagnosis of subjective cognitive decline (SCD) was established when a patient presented with cognitive complaints in the absence of objective cognitive, neurologic, or psychiatric impairment. ${ }^{17}$ Due to positive $\mathrm{AD}$ biomarkers and according to NIA-AA nomenclature, ${ }^{18}$ these participants were classified as preclinical $\mathrm{AD}$. Participants were included based on (1) diagnosis of probable $\mathrm{AD},{ }^{15} \mathrm{MCI}$ due to $\mathrm{AD},{ }^{16}$ or $\mathrm{SCD}^{17}$; (2) positive CSF biomarkers for $\mathrm{AD}$ (i.e., $\beta$-amyloid $[\mathrm{A} \beta]_{42}$ $<638 \mathrm{ng} / \mathrm{L}$ or tau/A $\beta_{42}$ fraction $>0.52^{19}$ ) or a positive $\mathrm{A} \beta \quad\left({ }^{18} \mathrm{~F}\right.$-flutemetamol, ${ }^{18} \mathrm{~F}$-florbetaben, ${ }^{18} \mathrm{~F}$-florbetapir, or $\left[{ }^{11} \mathrm{C}\right]$ Pittsburgh compound B) PET scan by visual assessment $^{20}$; (3) availability of a 3T T1-weighted structural MRI scan; and (4) Mini-Mental State Examination (MMSE) $\geq 10$. Exclusion criteria were (1) significant cerebrovascular disease on MRI, (2) a history of substance abuse, (3) major traumatic brain injury, (4) major psychiatric or neurologic disorders (other than $\mathrm{AD}$ ), and (5) meeting core clinical criteria for an atypical variant of $\mathrm{AD}$ (e.g., posterior cortical atrophy; figure 1).

\section{Standard protocol approvals, registrations, and patient consents}

Informed consent was obtained from all participants and the medical ethics review committee of the VU University Medical Center approved the study.

Figure 1 Flowchart of the sample selection

Participants selected based on

inclusion criteria $(\mathrm{N}=780)$ :

- Diagnosis of subjective cognitive decline, mild cognitive impairment, or probable $A D$ dementia

- Amyloid- $\beta$ positive (CSF or PET)

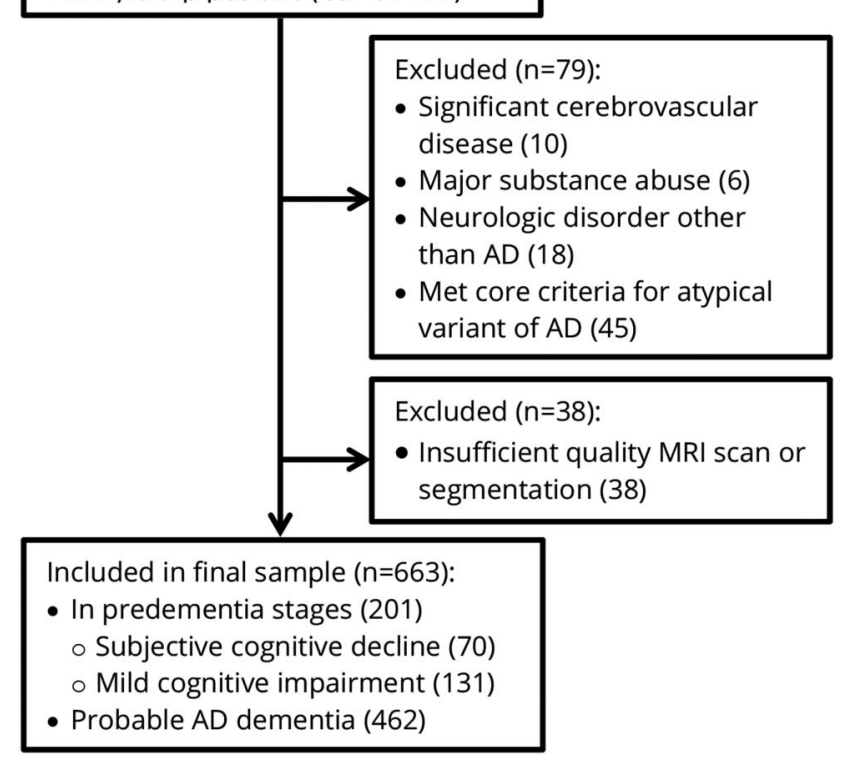

$A D=$ Alzheimer disease. 


\section{MRI}

All participants underwent MRI scans on a $3 \mathrm{~T}$ MRI scanner, according to standardized acquisition protocols including a T1 sequence. Three different scanner types were used: SignaHDxt $3 \mathrm{~T}(\mathrm{n}=493$, GE Healthcare [Cleveland, $\mathrm{OH}$ ], voxel size $0.94 \times 0.94 \times 1 \mathrm{~mm}$, echo time 3 milliseconds, repetition time 7.8 milliseconds, flip angle $12^{\circ}$, field of view $\left.240 \mathrm{~mm}\right)$, Vantage Titan $3 \mathrm{~T}(\mathrm{n}=105$, Toshiba Medical Systems [Glen Mills, PA], voxel size $1 \times 1$ $\times 1 \mathrm{~mm}$, echo time 3.2 milliseconds, repetition time 9.5 milliseconds, flip angle $7^{\circ}$, field of view $256 \mathrm{~mm}$ ), or Ingenuity TF PET-MRI 3T ( $\mathrm{n}=65$, Philips Medical Systems [Best, the Netherlands], voxel size $0.87 \times 0.87 \times 1 \mathrm{~mm}$, echo time 3 milliseconds, repetition time 7 milliseconds, flip angle $12^{\circ}$, field of view $250 \mathrm{~mm}$ ). All statistical models included scanner type as a covariate.

\section{CR and $B R$}

As a proxy of $C R$, we used the Verhage system ${ }^{21}$ to measure education. This is a standardized index (range 1-7), with a score of 1 indicating that primary school was not completed, while a score of 7 corresponds to an academic degree. ICV was used as a proxy measure of $\mathrm{BR}$ and was obtained by segmenting T1-weighted MRI using Statistical Parametric Mapping 12 software (SPM12; Wellcome Trust Centre for Neuroimaging, Institute of Neurology at University College, London, UK). This yields volumetric measures of gray matter, white matter, and CSF, which were summed to provide ICV.

\section{Neurodegeneration}

The concepts of CR and BR posit to explain discrepancies between observed and expected performance based on the level of underlying neuropathology. Therefore, operationalizations of CR and BR should include a measure of neuropathology. ${ }^{22}$ In the present study, we used whole brain gray matter volume relative to ICV (reflecting cerebral atrophy) as a surrogate measure of neuropathology.

\section{Cognition}

A standardized neuropsychological test battery was used to assess performance in 5 cognitive domains: memory (visual association test; Rey Auditory Verbal Learning Test immediate and delayed recall), attention (digit span forward; TrailMaking Test part A; Stroop test form I and II), executive functioning (frontal assessment battery; Stroop test form III; digit span backward), language (category fluency [animal naming]; naming condition of the visual association test), and visuospatial ability (number location; dot counting; fragmented letters). ${ }^{23,24}$ To obtain cognitive domain scores, all raw test scores were first converted into $Z$ scores using the mean and SD of equivalent neuropsychological test scores from an independent reference group of healthy controls $(\mathrm{n}=$ 533 , age $=59.7 \pm 9.8$ years, $46 \%$ male, MMSE $=28.9 \pm 1.0)$ of AD biomarker-negative participants with $S C D$. $Z$ scores for Trail-Making Test and Stroop test were inverted as higher scores indicate worse performance. $Z$ scores were combined into cognitive domain scores by averaging scores across tests within each domain. Composite scores for each cognitive domain were only calculated if there were data available on $\geq 2$ tests within that specific domain; otherwise that domain score was classified as missing ( $\mathrm{n}$ for missing domain scores: memory $=7$, attention $=7$, executive functioning $=10$, language $=25$, visuospatial ability $=26$ ). In addition, MMSE scores (available for all participants) were used as an index of global cognitive functioning.

\section{Statistical analysis}

We used multiple linear regression models, adjusted for cerebral atrophy, age, sex, and scanner type, to examine the effects of education and ICV on cognition. Cognitive domain $Z$ scores and MMSE scores were the dependent variables in the models and cases with missing cognitive domain scores were excluded from the analyses. First, we assessed the predictive effects of education and ICV separately (model 1), followed by a model including both predictors (model 2) to examine their independent effects. Next, we examined whether the effects of education and ICV on cognition differed according to disease stage, by performing regression models in predementia participants (SCD or MCI, $n$ $=201$ ) and in participants with dementia (probable $\mathrm{AD}, \mathrm{n}=$ 462). Differences in effects across disease stages were assessed by Welch $t$ tests, $^{25}$ using the regression slopes $(\beta)$ and corresponding standard error. ${ }^{26,27}$ To test the assumptions of the regression analyses, we plotted and checked residuals of all models. Residuals were normally distributed, heteroscedasticity was in conformance with test assumptions, and Durbin-Watson test statistics indicated independence of observations. Furthermore, variance inflation factor values, tolerance values, and correlations between variables did not indicate multicollinearity between predictors. Next, we dichotomized the total sample according to low vs high CR using a median split for education (Verhage $1-5=$ low education, $6-7=$ high education) and according to low vs high BR using a mean split for ICV $(1.12-1.51=$ low ICV, 1.52-2.01 = high ICV). Using these dichotomized groups, we computed a 4-level variable representing degree of total reserve; low $\mathrm{CR}$ and $\mathrm{BR}(\mathrm{CR}-/ \mathrm{BR}-, \mathrm{n}=$ 220), low $C R$ and high $B R(C R-/ B R+, n=180)$, high $C R$ and low $\mathrm{BR}(\mathrm{CR}+/ \mathrm{BR}-, \mathrm{n}=121)$, high $\mathrm{CR}$ and $\mathrm{BR}(\mathrm{CR}+/ \mathrm{BR}+, \mathrm{n}=$ 142). To assess differences in cognition across these 4 levels, we fitted general linear models, adjusted for cerebral atrophy, age, sex, and scanner type, and examined post hoc linear trends across levels. All statistical analyses were performed in SPSS version 20 (released 2011, IBM SPSS Statistics for Windows, Armonk, NY) and statistical significance in all models was set at $\alpha=0.05$ (2-tailed), uncorrected for multiple comparisons. GraphPad Prism (GraphPad Software, La Jolla, CA) version 6.0 was used for the figures.

\section{Results}

Demographic and clinical characteristics of the total sample and according to disease stage are presented in table 1 . There were no differences according to disease stage in sex $(p=0.17)$, age $(p=0.41)$, or ICV $(p=0.18)$, while education 
was lower in participants with dementia than in predementia participants $(p<0.05)$. As expected, participants with dementia had lower cognitive scores $(p<0.05)$ and reduced $\mathrm{ICV}$-corrected gray matter volumes (i.e., more cerebral atrophy, $p<0.05)$ compared to predementia participants. Pearson correlation analysis revealed a modest association between education and ICV in the total sample $(r=0.17, p<0.01)$. Furthermore, ICV-corrected gray matter volume was moderately associated with cognition (memory: $r=0.31$, attention: $r=0.36$, executive functioning: $r=0.41$, language: $r=0.31$, visuospatial ability: $r=0.39$, and MMSE: $r=0.45$, all $p<0.01$ ), adjusted for age, sex, and scanner type.

\section{Effects of CR and BR on cognition}

Multiple regression analyses with adjustment for cerebral atrophy, age, sex, and scanner type (model 1) revealed positive effects of both education and ICV on all cognitive domains (all $p<0.05$; table 2). When combining education and ICV in a single model (model 2), all effects survived, except for the effect of ICV on language ( $p=0.11$; table 2$)$. These results indicate that, while controlling for the degree of cerebral atrophy, both $\mathrm{CR}$ and $\mathrm{BR}$ have a positive effect on cognition in participants with positive $\mathrm{AD}$ biomarkers.

\section{Effects of CR and BR on cognition according to disease stage}

Next, we stratified the sample according to disease stage (dementia vs predementia) and performed model 1 and 2 in both patient groups. Model 1 showed positive effects of education on attention, executive functioning, and MMSE scores in predementia participants, and on memory, attention, executive functioning, visuospatial ability, and MMSE in participants with dementia (all $p<0.05$ ). Furthermore, there were positive effects of ICV on executive functioning and MMSE in predementia participants $(p<0.05)$ and on memory, attention, executive functioning, visuospatial ability, and MMSE in participants with dementia (all $p<0.05$; table 2 ). When combining education and ICV in one model (model 2 ), we found that all effects of education and ICV survived $(p<0.05)$, with the exception of the effects on memory in participants with dementia ( $p=0.08$ for education, $p=0.11$ for ICV; table 2$)$ and the effect of ICV on MMSE in predementia participants $(p=$ 0.07 ; table 2 ). The effect sizes of education on attention ( $\beta=$ $0.39, p<0.01$ vs $\beta=0.21, p<0.01)$ and executive functioning ( $\beta=0.46, p<0.05$ vs $\beta=0.26, p<0.01$ ) were $46 \%$ and $43 \%$ larger in predementia participants than in participants with dementia (Welch $t=2.40, p<0.01$ and $t=2.83$, $p<0.01$; figure 2A). This indicates that high CR is especially beneficial for cognition in early stages of $\mathrm{AD}$. There were no differences for the effects of ICV (i.e., BR) according to disease stage (figure 2B).

\section{Differences in cognitive functioning across levels of reserve}

Subsequently, we constructed a 4-level variable (i.e., $\mathrm{CR}-/ \mathrm{BR}-, \mathrm{CR}-/ \mathrm{BR}+, \mathrm{CR}+/ \mathrm{BR}-$, and $\mathrm{CR}+/ \mathrm{BR}+$ ) and fitted general linear models, adjusted for cerebral atrophy, age, sex, and scanner type, to assess cognitive performance across

Table 1 Demographic and clinical characteristics of the total sample and according to disease stage

\begin{tabular}{|c|c|c|c|}
\hline & Total $(n=663)$ & Predementia $(n=201)$ & Dementia $(n=462)$ \\
\hline Diagnosis & & SCD (70); MCI (131) & Probable AD (462) \\
\hline Sex, \% male & 49 & 53 & 47 \\
\hline Age, y & $66.2(7.4)$ & $66.6(7.5)$ & $66.1(7.4)$ \\
\hline Education, Verhage, median (range) & $5(2-7)$ & $5(2-7)^{a}$ & $5(2-7)$ \\
\hline ICV & $1.51(0.16)$ & $1.52(0.16)$ & $1.50(0.16)$ \\
\hline MMSE & $22.7(4.8)$ & $27.0(2.2)^{\mathrm{a}}$ & $20.8(4.3)$ \\
\hline Cerebral atrophy ${ }^{\mathbf{b}}$ & $0.39(0.04)$ & $0.41(0.04)^{\mathrm{a}}$ & $0.38(0.04)$ \\
\hline \multicolumn{4}{|l|}{ Cognitive function $Z$ scores $^{c}$} \\
\hline Memory & $-3.78(3.27)$ & $-1.39(1.56)^{\mathrm{a}}$ & $-4.83(3.36)$ \\
\hline Attention & $-1.87(2.58)$ & $-0.39(0.81)^{a}$ & $-2.53(2.81)$ \\
\hline Executive functioning & $-1.84(1.81)$ & $-0.50(0.91)^{a}$ & $-2.43(1.80)$ \\
\hline Language & $-1.09(1.33)$ & $-0.29(0.58)^{a}$ & $-1.45(1.41)$ \\
\hline Visuospatial ability & $-1.69(2.67)$ & $-0.21(0.87)^{a}$ & $-2.35(2.93)$ \\
\hline
\end{tabular}

Abbreviations: $A D=$ Alzheimer disease; $I C V=$ intracranial volume in dm ${ }^{3} ; \mathrm{MCI}=$ mild cognitive impairment; $\mathrm{MMSE}=\mathrm{Mini}-\mathrm{Mental}$ State Examination; SCD = subjective cognitive decline.

Values are depicted as mean (SD) unless otherwise indicated. Group comparisons were performed using $\chi^{2}$, Mann-Whitney $U$, or independent samples $t$ tests, where appropriate.

a Predementia > dementia.

${ }^{\mathrm{b}}$ Gray matter volumes relative to ICV; lower values indicate more cerebral atrophy.

c $Z$ scores calculated using the mean and SD of independent reference group. 
Table 2 Effects of education and intracranial volume (ICV) in the total sample and according to disease stage

\begin{tabular}{|c|c|c|c|c|c|c|c|c|}
\hline \multirow[b]{2}{*}{ Domain } & & \multicolumn{3}{|c|}{ Education } & & \multicolumn{3}{|c|}{ ICV } \\
\hline & & \multicolumn{2}{|l|}{ Model $1^{a}$} & \multicolumn{2}{|l|}{ Model $2^{b}$} & \multicolumn{2}{|l|}{ Model $1^{\mathrm{a}}$} & Model $2^{\circ}$ \\
\hline \multicolumn{2}{|l|}{ Memory } & \multicolumn{2}{|l|}{$0.12^{d}$} & \multicolumn{2}{|l|}{$0.10^{d}$} & \multicolumn{2}{|l|}{$0.18^{d}$} & $0.16^{d}$ \\
\hline \multicolumn{2}{|l|}{ Attention } & \multicolumn{2}{|l|}{$0.24^{d}$} & \multicolumn{2}{|l|}{$0.22^{d}$} & \multicolumn{2}{|l|}{$0.21^{d}$} & $0.15^{d}$ \\
\hline \multicolumn{2}{|l|}{ Executive function } & \multicolumn{2}{|l|}{$0.31^{d}$} & \multicolumn{2}{|l|}{$0.28^{d}$} & \multicolumn{2}{|l|}{$0.26^{d}$} & $0.19^{d}$ \\
\hline \multicolumn{2}{|l|}{ Language } & \multicolumn{2}{|l|}{$0.11^{d}$} & \multicolumn{2}{|l|}{$0.10^{d}$} & \multicolumn{2}{|l|}{$0.11^{d}$} & 0.08 \\
\hline \multicolumn{2}{|l|}{ Visuospatial ability } & \multicolumn{2}{|l|}{$0.16^{d}$} & \multicolumn{2}{|l|}{$0.14^{d}$} & \multicolumn{2}{|l|}{$0.18^{d}$} & $0.15^{d}$ \\
\hline \multirow[t]{3}{*}{ MMSE } & & \multicolumn{2}{|l|}{$0.28^{d}$} & \multicolumn{2}{|l|}{$0.25^{d}$} & \multicolumn{2}{|l|}{$0.26^{\mathrm{d}}$} & $0.19^{d}$ \\
\hline & & \multicolumn{3}{|c|}{ Education } & \multicolumn{4}{|c|}{ ICV } \\
\hline & \multicolumn{2}{|c|}{ Predementia $(n=201)$} & \multicolumn{2}{|c|}{ Dementia $(n=462)$} & \multicolumn{2}{|c|}{ Predementia $(n=201)$} & \multicolumn{2}{|c|}{ Dementia $(n=462)$} \\
\hline Domain & Model $1^{a}$ & Model $2^{b}$ & Model $1^{a}$ & Model $2^{b}$ & Model $1^{a}$ & Model $2^{c}$ & Model $1^{a}$ & Model $2^{c}$ \\
\hline Memory & 0.04 & 0.02 & $0.10^{d}$ & 0.08 & 0.12 & 0.12 & $0.13^{d}$ & 0.10 \\
\hline Attention & $0.40^{d}$ & $0.39^{d}$ & $0.23^{d}$ & $0.21^{d}$ & 0.12 & 0.06 & $0.21^{d}$ & $0.14^{d}$ \\
\hline Executive function & $0.48^{d}$ & $0.46^{d}$ & $0.28^{d}$ & $0.26^{d}$ & $0.26^{d}$ & $0.18^{d}$ & $0.23^{d}$ & $0.15^{d}$ \\
\hline Language & 0.13 & 0.13 & 0.06 & 0.06 & -0.01 & -0.03 & 0.08 & 0.05 \\
\hline Visuospatial ability & 0.10 & 0.08 & $0.16^{d}$ & $0.14^{d}$ & 0.15 & 0.14 & $0.17^{d}$ & $0.13^{d}$ \\
\hline MMSE & $0.34^{d}$ & $0.32^{d}$ & $0.27^{d}$ & $0.25^{d}$ & $0.21^{d}$ & 0.16 & $0.23^{d}$ & $0.15^{d}$ \\
\hline
\end{tabular}

Abbreviations: ICV = intracranial volume; MMSE = Mini-Mental State Examination.

Values depicted are partial regression coefficients $(\beta)$.

a Effects adjusted for cerebral atrophy, age, sex, and scanner type.

${ }^{b}$ Effects adjusted for cerebral atrophy, age, sex, scanner type, and intracranial volume.

' Effects adjusted for cerebral atrophy, age, sex, scanner type, and education.

dignificant effect at $p<0.05$.

levels. We observed a linear trend across all cognitive domains and MMSE with highest estimated marginal means for $\mathrm{CR}+/ \mathrm{BR}+$, followed by $\mathrm{CR}+/ \mathrm{BR}-, \mathrm{CR}-/ \mathrm{BR}+$, and then CR-/BR- ( $p$ for trend $<0.05$, figure 3 ). Sensitivity analysis (switching the $\mathrm{CR}-/ \mathrm{BR}+$ and $\mathrm{CR}+/ \mathrm{BR}-$ groups) confirmed the linear trend $(p<0.05)$.

\section{Discussion}

The main findings of our study are (1) CR and BR both have independent positive effects on cognition in participants with biomarker evidence of $\mathrm{AD}$, adjusted for cerebral atrophy, (2) the effects of $\mathrm{CR}$ on attention and executive functioning were greater in predementia participants than in participants with dementia, (3) the effects of CR were generally greater than those of BR, and (4) there was a linear trend for better cognitive performance in all domains (adjusted for cerebral atrophy) in the $\mathrm{CR}+\mathrm{BR}+$ group, followed by $\mathrm{CR}+\mathrm{BR}-$, $\mathrm{CR}-/ \mathrm{BR}+$, and then $\mathrm{CR}-/ \mathrm{BR}-$.

The positive effects of $\mathrm{CR}$ and $\mathrm{BR}$ (as indicated by education and ICV) on cognitive functioning in this study are in line with most literature, suggesting that higher education and greater ICV positively influence the cognitive trajectory of patients with $\mathrm{AD}^{7-11} \mathrm{We}$ extend on these findings by demonstrating that $\mathrm{CR}$ and $\mathrm{BR}$ differentially mitigate cognitive symptoms in $\mathrm{AD}$, as $\mathrm{CR}$ was most beneficial in predementia stages (there was no disease stage-specific effect for $\mathrm{BR}$ ) and the effects of CR were overall stronger than those of $\mathrm{BR}$. Although CR and BR are related to similar underlying factors, our results thus indicate that they are at least partially separate components of a larger concept (i.e., reserve) rather than interchangeable terms describing a single entity. This is further highlighted by the small correlation $(r=0.17)$ between education and ICV in our sample.

Physiologic mechanisms underlying the protective effect of CR may include facilitating the development of new cognitive strategies, ${ }^{4}$ modulation of functional connectivity in hub regions such as the posterior cingulate cortex, ${ }^{28,29}$ and strengthened network reliability, ${ }^{30}$ which are all associated with higher education. These mechanisms actively support the brain to cope with neuropathology. The mechanism underlying $\mathrm{BR}$ is to increase resilience to neuropathology through greater quantities of premorbid brain parenchyma. In the event of neurodegeneration, the necessary structural integrity to maintain normal cognitive functioning will be retained for a longer period in individuals with high $\mathrm{BR}$ than in individuals with low $\mathrm{BR}^{4-6}$ More detailed examinations into 
Figure 2 Effect sizes of education and intracranial volume (ICV) on cognition according to disease stage

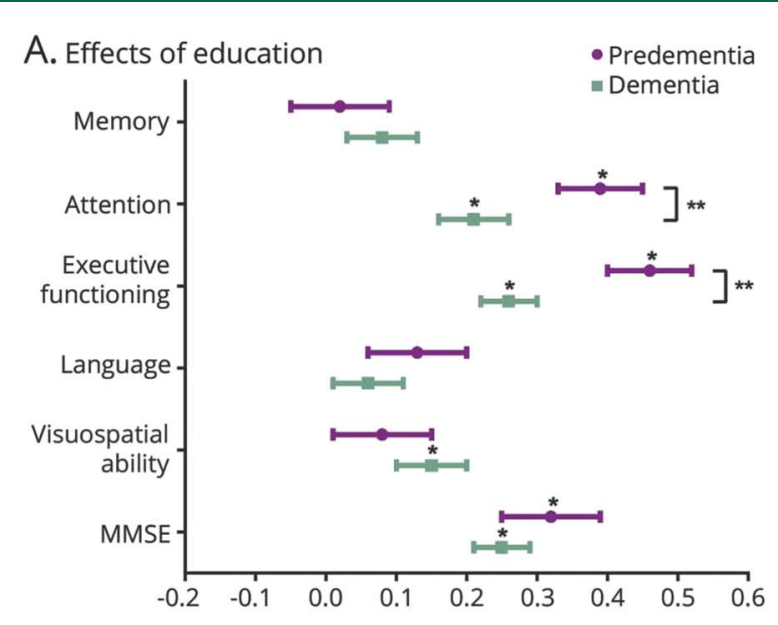

B. Effects of ICV

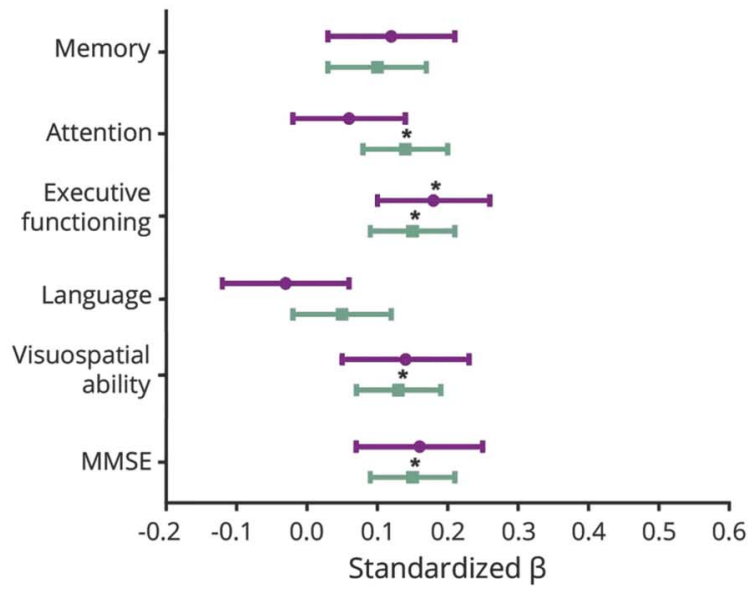

(A) Effect size of education. (B) Effect size of ICV. Effect sizes are partial regression coefficients $(\beta)$, adjusted for cerebral atrophy, age, sex, and scanner type. Error bars indicate the standard error. *Significant effect at $p<0.05$. ${ }^{*}$ Difference of effect sizes between groups (Welch $t$ test, $p<0.05)$. MMSE $=$ Mini-Mental State Examination.

the effects of $\mathrm{BR}$, for instance focused on microstructural integrity of the brain (e.g., synaptic density measured with $\mathrm{PET}^{31}$ ), could provide a more comprehensive depiction of the mechanisms behind the protective effect of $\mathrm{BR}$ in $\mathrm{AD}$.

A considerable body of literature has described positive effects of education and ICV on cognition, but these effectsespecially for ICV - have not been replicated in all studies. ${ }^{32,33}$ Strengths of the present study, such as the large sample size, inclusion of $\mathrm{AD}$ biomarker-positive participants ranging from preclinical to dementia stages, availability of 3T MRI, SPM12based tissue segmentation, ${ }^{34}$ and detailed neuropsychological testing, likely increased sensitivity for detecting effects of education and ICV compared to some previous studies. Some limitations of the present study also need to be addressed. First, there are inherent limitations related to cross-sectional designs and longitudinal follow-up studies are needed to confirm whether reserve has a direct effect on the progression
Figure 3 Standardized cognitive domain and Mini-Mental State Examination (MMSE) scores (adjusted for cerebral atrophy) across degrees of total reserve

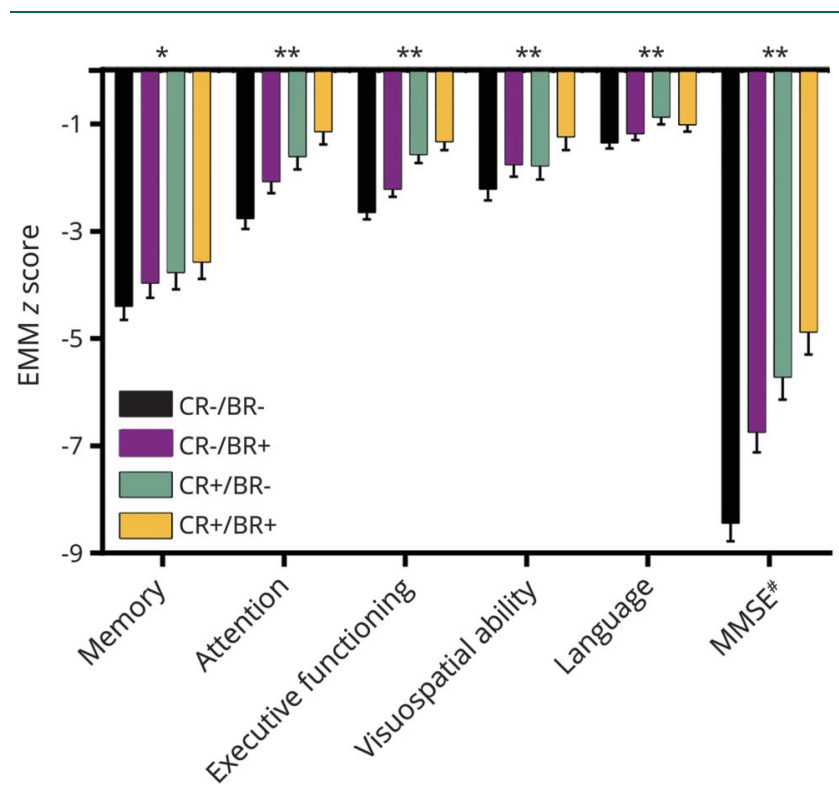

Data are estimated marginal means (EMM) (plus standard error) for cognitive domain $Z$ scores, adjusted for cerebral atrophy, age, sex, and scanner type. $\mathrm{BR}=$ brain reserve; $\mathrm{CR}=$ cognitive reserve. ${ }^{\star} p$ for trend $<0.05$. ${ }^{* *} p$ for trend $<0.01$. \#MMSE scores were converted to $Z$ scores for visualization purposes; we used raw scores in the statistical analyses.

of cognitive decline. Second, the difference in sample size between the predementia group $(\mathrm{n}=201)$ and dementia group $(n=462)$ may have resulted in more significant effects being observed in the dementia group with similar effect sizes. However, power analyses revealed that both sample sizes were sufficient to detect effects and interpretation of results was focused on (differences in) effect sizes rather than levels of significance. Possible associations between predictors, especially between ICV and cerebral atrophy, may have resulted in multicollinearity in the regression models. However, we conducted thorough assessment of multicollinearity by examining tolerance values and variance inflation factors, and these assessments revealed no indication for significant multicollinearity in the regression models. Furthermore, the modest correlations between predictors $(r=0.32$ between ICV and cerebral atrophy in the total sample) fall well below the assumption that a correlation higher than $r=0.70$ indicates multicollinearity. ${ }^{35}$ Third, our relatively young cohort (mean age $66.2 \pm 7.4$ years) may be characterized by an overrepresentation of hippocampal-sparing $\mathrm{AD}^{36}$ and relative paucity of comorbidities. This should be taken into account when generalizing or replicating our findings to cohorts with a higher average age. Finally, reserve is a hypothetical construct that is often measured using proxies, which come with inherent limitations and imperfections. For instance, there may be geographical differences related to access and level of education and there exists a range of methods to measure education, from total years of school to categorical scales such 
as the Verhage scale. ${ }^{21}$ Also, education is associated with other socioeconomic characteristics (e.g., occupation, access to general health care), which in turn may affect CR. Furthermore, ICV serves as an easily obtainable proxy of BR but may reflect early childhood brain development to a higher extent than later childhood and adolescent influences.

Our results indicate that $\mathrm{CR}$, as measured by education, has the greatest potential to delay or slow down cognitive decline in $\mathrm{AD}$. This highlights the importance of education in early life. However, our findings regarding the differential effects of CR between disease stages may also serve tailoring clinical interventions in late life. We have shown that the effects of $\mathrm{CR}$ are especially beneficial in the earlier phases of the disease, which indicates that interventions (e.g., physical activity interventions ${ }^{37}$ or cognitive training) would preferentially be offered early on in the disease course. BR as measured by ICV is in itself a nonmodifiable factor. However, our results regarding the additive effects of CR and BR suggest that interventions tailored to increasing $C R$ would show maximized treatment effects in individuals with high BR. These insights may help to tailor interventions and to reduce the rate of cognitive decline in neurodegenerative diseases and promote successful aging.

\section{Author contributions}

Colin Groot contributed to study design; acquisition, analysis, and interpretation of data; writing and revising the manuscript; and performed statistical analysis. Anna C. van Loenhoud contributed to study design and statistical analysis and helped with interpretation of data and revising the manuscript. Bart N.M. van Berckel was involved in the acquisition of PET data and revised the manuscript. Philip Scheltens and Frederik Barkhof helped with critical revision of manuscript for intellectual content and interpretation of data. Teddy Koene was involved in the acquisition of neuropsychology data. Charlotte C. Teunissen was involved in acquisition of CSF biomarker data and helped with critical revision of manuscript for intellectual content and interpretation of data. Wiesje M. van der Flier contributed to acquisition of patient data from the Amsterdam Dementia Cohort, performed statistical analysis, and helped with interpretation of results and critical revision of manuscript for intellectual content. Rik Ossenkoppele contributed to study concept and design and critical revision of manuscript for intellectual content; helped in writing and revising the manuscript, statistical analysis, acquisition and analysis of MRI data, and revising the manuscript; and supervised the study.

\section{Study funding}

Research of the VUMC Alzheimer Center is part of the Neurodegeneration Research Program of the Neuroscience Campus Amsterdam. The VUMC Alzheimer Center is supported by Alzheimer Nederland and Stichting VUMC funds. The clinical database structure was developed with funding from Stichting Dioraphte. This research was funded by the Internationale Stichting Alzheimer Onderzoek (ISAO) (to
R.O.); Marie Curie FP7 International Outgoing Fellowship (628812) (to R.O.); and the donors of Alzheimer's Disease Research, a program of BrightFocus Foundation (to R.O.). F.B. is supported by the NIHR UCLH biomedical research center.

\section{Disclosure}

C. Groot and A. van Loenhoud report no disclosures relevant to the manuscript. F. Barkhof is editorial board member of Brain, European Radiology, Neurology ${ }^{\circledR}$, Multiple Sclerosis Journal, and Radiology; performed consultancy and received personal compensation and honoraria from Bayer-Schering Pharma and Genzyme; received compensation (personal and to institution) and honoraria from Biogen-IDEC, TEVA, Merck-Serono, Novartis, Roche, Synthon BV, and Jansen Research; received payment for development of educational presentations from IXICO and Biogen-IDEC (to institution); is funded by a Dutch MS Society grant, EU-FP7/H2020; and is supported by the NIH Research Biomedical Research Center at University College London Hospital. B. van Berckel, T. Koene, C. Teunissen, P. Scheltens, W. van der Flier, and R. Ossenkoppele report no disclosures relevant to the manuscript. Go to Neurology.org/ $\mathrm{N}$ for full disclosures.

Received June 16, 2017. Accepted in final form September 27, 2017.

\section{References}

1. Katzman R, Terry R, DeTeresa R, et al. Clinical, pathological, and neurochemical changes in dementia: a subgroup with preserved mental status and numerous neocortical plaques. Ann Neurol 1988;23:138-144.

2. Yaffe K, Weston A, Graff-Radford NR, et al. Association of plasma beta-amyloid level and cognitive reserve with subsequent cognitive decline. JAMA 2011;305:261-266.

3. Kemppainen NM, Aalto S, Karrasch M, et al. Cognitive reserve hypothesis: Pittsburgh compound $\mathrm{B}$ and fluorodeoxyglucose positron emission tomography in relation to education in mild Alzheimer's disease. Ann Neurol 2008;63:112-118.

4. Stern Y. What is cognitive reserve? Theory and research application of the reserve concept. J Int Neuropsychol Soc 2002;8:448-460.

5. Stern Y. Cognitive reserve in ageing and Alzheimer's disease. Lancet Neurol 2012;11: 1006-1012.

6. Stern Y. Cognitive reserve. Neuropsychologia 2009;47:2015-2028

7. Liu Y, Julkunen V, Paajanen T, et al. Education increases reserve against Alzheimer's disease: evidence from structural MRI analysis. Neuroradiology 2012;54:929-938.

8. Perneczky R, Wagenpfeil S, Lunetta KL, et al. Education attenuates the effect of medial temporal lobe atrophy on cognitive function in Alzheimer's disease: the MIRAGE study. J Alzheimers Dis 2009; 17:855-862.

9. Guo LH, Alexopoulos P, Wagenpfeil S, Kurz A, Perneczky R, Initiative ADN. Brain size and the compensation of Alzheimer's disease symptoms: a longitudinal cohort study. Alzheimers Dement 2013;9:580-586.

10. Negash S, Xie S, Davatzikos C, et al. Cognitive and functional resilience despite molecular evidence of Alzheimer's disease pathology. Alzheimers Dement 2013;9: 89-95.

11. Wolf H, Julin P, Gertz HJ, Winblad B, Wahlund LO. Intracranial volume in mild cognitive impairment, Alzheimer's disease and vascular dementia: evidence for brain reserve? Int J Geriatr Psychiatry 2004;19:995-1007.

12. Sperber GH. Transitions in craniofacial biology: a tribute to Bernard G. Sarnat. J Craniofac Surg 2012;23:124-125.

13. Courchesne E, Chisum HJ, Townsend J, et al. Normal brain development and aging: quantitative analysis at in vivo MR imaging in healthy volunteers. Radiology 2000; 216:672-682.

14. van der Flier WM, Pijnenburg YAL, Prins N, et al. Optimizing patient care and research: the Amsterdam Dementia Cohort. J Alzheimers Dis 2014;41:313-327.

15. McKhann GM, Knopman DS, Chertkow H, et al. The diagnosis of dementia due to Alzheimer's disease: recommendations from the National Institute on AgingAlzheimer's Association workgroups on diagnostic guidelines for Alzheimer's disease. Alzheimers Dement 2011;7:263-269.

16. Albert MS, DeKosky ST, Dickson D, et al. The diagnosis of mild cognitive impairment due to Alzheimer's disease: recommendations from the National Institute on AgingAlzheimer's Association workgroups on diagnostic guidelines for Alzheimer's disease. Alzheimers Dement 2011;7:270-279. 
17. Jessen F, Amariglio RE, van Boxtel M, et al. A conceptual framework for research on subjective cognitive decline in preclinical Alzheimer's disease. Alzheimers Dement 2014;10:844-852.

18. Sperling RA, Aisen PS, Beckett LA, et al. Toward defining the preclinical stages of Alzheimer's disease: recommendations from the National Institute on AgingAlzheimer's Association workgroups on diagnostic guidelines for Alzheimer's disease. Alzheimers Dement 2011;7:280-292.

19. Zwan M, van Harten A, Ossenkoppele R, et al. Concordance between cerebrospinal fluid biomarkers and [ ${ }^{11}$ C $]$ PIB PET in a memory clinic cohort. J Alzheimers Dis 2014;41:801-807.

20. Ossenkoppele R, Pijnenburg YAL, Perry DC, et al. The behavioural/dysexecutive variant of Alzheimer's disease: clinical, neuroimaging and pathological features. Brain 2015; 138:2732-2749.

21. Verhage F. Intelligence and Age: Study With Dutch People Aged 12-77. Assen: van Gorcum; 1964

22. Jones RN, Manly J, Glymour MM, Rentz DM, Jefferson AL, Stern Y. Conceptual and measurement challenges in research on cognitive reserve. J Int Neuropsychol Soc 2011;17:593-601.

23. Ossenkoppele R, Zwan MD, Tolboom N, et al. Amyloid burden and metabolic function in early-onset Alzheimer's disease: parietal lobe involvement. Brain 2012; 135:2115-2125.

24. van Loenhoud AC, Wink AM, Groot C, et al. A neuroimaging approach to capture cognitive reserve: application to Alzheimer's disease. Hum Brain Mapp 2017;38: 4703-4715.

25. Welch BL. On the comparison of several mean values: an alternative approach. Biometrika 1951;38:330.
26. Paternoster R, Brame R. Using the correct statistical test for the equality of regression coefficients. Criminology 1998;36:859-866.

27. Clogg CC, Petkova E, Haritou A. Statistical methods for comparing regression coefficients between models. Am J Sociol 1995;100:1261-1293.

28. Bozzali M, Dowling C, Serra L, et al. The impact of cognitive reserve on brain functional connectivity in Alzheimer's disease. J Alzheimers Dis 2015;44:243-250.

29. Stern Y, Habeck C, Moeller J, et al. Brain networks associated with cognitive reserve in healthy young and old adults. Cereb Cortex 2005;15:394-402.

30. Wook Yoo S, Han CE, Shin JS, et al. A network flow-based analysis of cognitive reserve in normal ageing and Alzheimer's disease. Sci Rep 2015;5:10057.

31. Finnema SJ, Nabulsi NB, Eid T, et al. Imaging synaptic density in the living human brain. Sci Transl Med 2016;8:348-396.

32. Tate DF, Neeley ES, Norton MC, et al. Intracranial volume and dementia: some evidence in support of the cerebral reserve hypothesis. Brain Res 2011;1385:151-162.

33. Jenkins R, Fox NC, Rossor AM, Harvey RJ, Rossor MN. Intracranial volume and Alzheimer disease: evidence against the cerebral reserve hypothesis. Arch Neurol 2000;57:220-224.

34. Malone IB, Leung KK, Clegg S, et al. Accurate automatic estimation of total intracranial volume: a nuisance variable with less nuisance. Neuroimage 2015;104:366-372.

35. Dormann CF, Elith J, Bacher S, et al. Collinearity: a review of methods to deal with it and a simulation study evaluating their performance. Ecography 2013;36:27-46.

36 Murray ME, Graff-Radford NR, Ross OA, et al. Neuropathologically defined subtypes of Alzheimer's disease with distinct clinical characteristics: a retrospective study. Lancet Neurol 2011;10:785-796.

37. Groot C, Hooghiemstra AM, Raijmakers PGHM, et al. The effect of physical activity on cognitive function in patients with dementia: a meta-analysis of randomized control trials. Ageing Res Rev 2016;25:13-23. 


\section{Differential effects of cognitive reserve and brain reserve on cognition in Alzheimer disease}

Colin Groot, MSc, Anna C. van Loenhoud, MSc, Frederik Barkhof, MD, PhD, Bart N.M. van Berckel, MD, PhD, Teddy Koene, MSc, Charlotte C. Teunissen, MD, PhD, Philip Scheltens, MD, PhD, Wiesje M. van der Flier, PhD, and Rik Ossenkoppele, PhD

Neurology ${ }^{\circledR}$ 2018;90:64. doi:10.1212/WNL.0000000000004802

\section{Study question}

What are the cross-sectional effects of cognitive reserve (CR) and brain reserve (BR) on cognition across the spectrum of Alzheimer disease $(\mathrm{AD})$ ?

\section{Summary answer}

$\mathrm{CR}$ and BR both independently and additively mitigate cognitive symptom severities in $\mathrm{AD}$, with the benefits of $\mathrm{CR}$ being strongest in predementia stages.

\section{What is known and what this article adds}

$\mathrm{CR}$ and $\mathrm{BR}$ both mitigate the cognitive symptoms of $\mathrm{AD}$ by helping patients cope with neuropathologic changes. This study elucidates how CR and BR independently and additively affect various cognitive domains in different $\mathrm{AD}$ stages.

\section{Participants and setting}

The study included 462 persons with dementia-stage $\mathrm{AD}$ and 201 persons with predementia $\mathrm{AD}$. They were selected from the Amsterdam Dementia Cohort and had visited the VU University Medical Center Amsterdam between January 2008 and December 2015.

\section{Design, size, and duration}

The study used education levels, as measured with the Verhage system, as a proxy for CR and intracranial volume (ICV), as measured with MRI, as a proxy for BR. The ratio of whole-brain gray matter volume to ICV was used as a proxy for cerebral atrophy.

\section{Primary outcomes}

The primary outcomes were scores in 5 cognition domains: memory, attention, visuospatial, language, and executive functions.

\section{Main results and the role of chance}

Compared to patients in predementia stages, those with dementia had lower education, lower cognitive scores, and greater cerebral atrophy $(p<0.05$ for all). Multiple regression analyses showed that, controlling for the effects of cerebral atrophy, greater education and ICV had independent positive effects on all cognitive domains except for the absence of an effect of ICV

\author{
Correspondence \\ C. Groot \\ c.groot3@vumc.nl
}

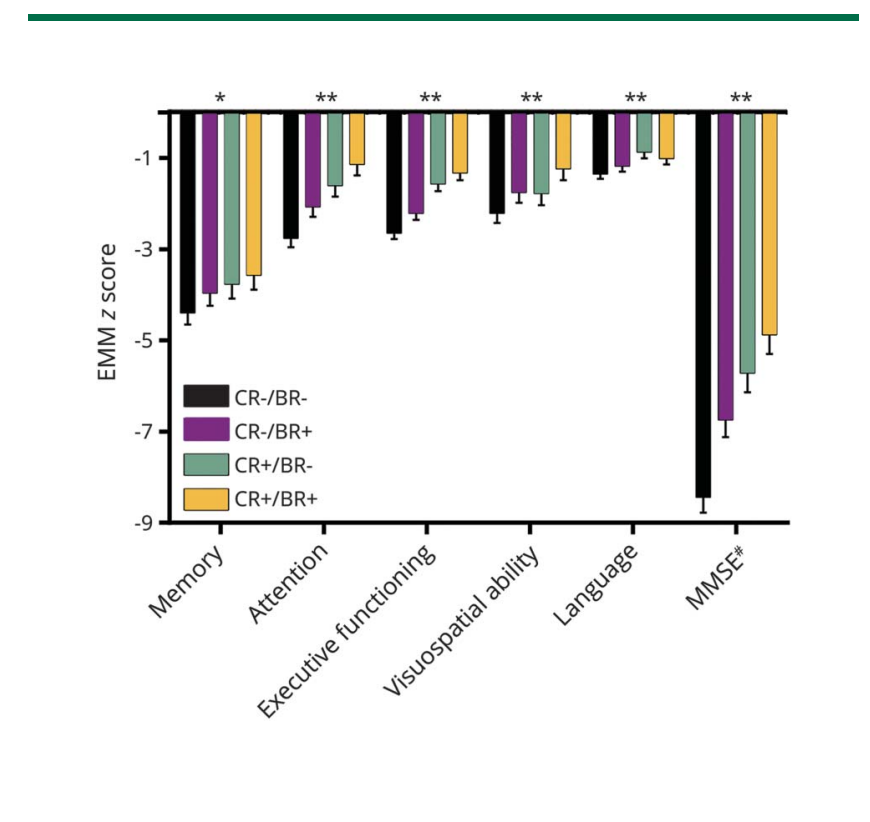

on language $(p<0.05)$. General linear models confirmed the additively beneficial effects of education and ICV on all domains ( $p<0.05$ for trend). Analyses by disease stage showed that the effects of CR were especially beneficial in predementia cases and were generally larger than the effects of $\mathrm{BR}$, but there was no benefit for education in patients with dementia.

\section{Bias, confounding, and other reasons for caution}

The cross-sectional design of this study precluded any determination of whether $\mathrm{CR}$ and $\mathrm{BR}$ directly affect the progression of cognitive decline. $\mathrm{CR}$ and $\mathrm{BR}$ were measured via proxies.

\section{Generalizability to other populations}

The participants were relatively young (mean age $66.2 \pm 7.4$ years), so there is limited generalization to older patients.

\section{Study funding/potential competing interests}

This study was funded by various medical research foundations. Dr. Barkhof has received personal compensation from various pharmaceutical companies and serves on several journals' editorial boards. Go to Neurology.org/N for full disclosures. 